\title{
Fatty acid, sterol and proximate compositions of peanut species (Arachis L.) seeds from Bolivia and Argentina
}

\author{
By Nelson R. Grosso'", Julio A. Zygadlo², Laura V. Burroni2 and Carlos A. Guzmán²
}

\author{
1Cátedra de Química Biológica, Facultad de Ciencias Agropecuarias, Universidad Nacional de Córdoba \\ (ICTA, CONICET), Avda. Valparaíso s/n., CC 509, 5000 Córdoba, Argentina. \\ 2 Instituto de Ciencias y Tecnología de los Alimentos (ICTA), FCEFyN-UNC, IMBIV-CONICET, \\ Avda. Velez Sarsfield 299, 5000 Córdoba, Argentina
}

\section{RESUMEN}

Ácidos grasos, esteroles y composiciones porcentuales de semillas de especies (Arachis L.) de cacahuete de Bolivia y Argentina

Los contenidos en aceite, proteína, ceniza e hidratos de carbono, índice de acidez, composiciones en ácidos grasos y esteroles fueron estudiadas en semillas de Arachis correntina, $A$. durannensis, A. Monticola, A. batizocoi, y A. cardenasii originaria de Bolivia y Argentina. El contenido en aceite fue mayor en A batizocoi (valor medio 53,35\%). El nivel de proteína fue más alto en $A$. monticola (valor medio $29,40 \%$ ) y $A$. durannensis $(29,13 \%)$. El valor medio del ácido oleico varió entre $34,91 \%(A$. durannensis y $A$. cardenasii) y $42,60 \%$ (Arachis correntina), y el ácido linoleíco osciló entre $40,23 \%$ (A. correntina) y $45,86 \%$ (A.durannensis). La mejor relación oleico a linoleico fue exhibida por $A$. correntina (1.06). El índice de iodo fue más bajo en $A$. batizocoi $(106,0)$. La composición esterólica en las diferentes especies de cacahuetes mostró la más alta concentración de B-sitosterol (los valores medios oscilaron entre $55,70-58,70 \%$ ) seguido por campesterol (15,18-16,47\%), estigmasterol (10,67-12,27\%) y $\Delta^{5}$-avenasterol $(10,80-12,13 \%)$.

PALABRAS-CLAVE: Ácido graso - Arachis L. - Argentina Bolivia - Cacahuete - Esterol.

\section{SUMMARY}

Fatty acid, sterol and proximate compositions of peanut species (Arachis L.) seeds from Bolivia and Argentina

The oil, protein, ash and carbohydrates contents, iodine value, fatty acid and sterol compositions were studied in seeds of Arachis correntina, A. durannensis, A. monticola, A. batizocoi, and $A$. cardenasii originating from Bolivia and Argentina. Oil content was greatest in $A$. batizocoi (mean value $53,35 \%$ ). The protein level was higher in $A$. monticola (mean value $29,40 \%$ ) and A. durannensis $(29,13 \%)$. Mean value of oleic acid varied between $34,91 \%$ ( $A$. durannensis and $A$. cardenasii) and $42,60 \%$ (Arachis correntina), and linoleic acid oscilated between $40,23 \%$ (A. correntina) and $45,86 \%$ (A. durannensis). The better oleic to linoleic ratio was exhibited by $A$. correntina $(1,06)$. lodine value was lower in $A$. batizocoi $(106,0)$. The sterol composition in the different peanut species showed higher concentration of Bsitosterol (mean values oscilated between 55,70-58,70\%) following by campesterol $(15,18-16,47 \%)$, stigmasterol $(10,67-$ $12,27 \%)$ and $\Delta^{5}$-avenasterol $(10,80-12,13 \%)$.

KEY-WORDS: Arachis L. - Argentine - Bolivia - Fatty acid Peanut - Sterol.

\section{INTRODUCTION}

At present there are 68 described wild species of Arachis native to South America (Krapovickas and Gregory, 1994). Substantial evidence suggests that the genetic base or gene pool of the cultivated peanut, $A$. hypogaea L., does not have the reserve germplasm needed to resist many of the new agricultural problems brought on by pollution, dwindling water supplies, and the necessity for biological control methods against insects and plant pathogens. Wild species of Arachis contain new sources of germplasm which can be used to increase variability in the genetic base of cultivated peanut (Stalker et al., 1989).

Leguminous seeds make an important contribution to the diet in many tropical countries. They are a good source of protein, lipid, and fatty acids for human nutrition (Gaydou et al., 1983). The fatty acid composition of endogenous fats plays and important role in determining shelf life, nutrition, and flavor of food products. The lipids and proteins of cultivated peanut seeds have been widely studied (Ahmed and Young, 1982). The objective of this work was to characterize some chemical components of wild species of Arachis.

\section{EXPERIMENTAL}

\section{Plant material}

Sound and mature seeds of Arachis correntina (Burkart) Krapov, \& W. C. Gregory, A. durannensis Krapov. \& W. C. Gregory, A. monticola Krapov. \& Rigoni, A. batizocoi Krapov. \& W. C. Gregory, and A. cardenasii Krapov. \& W. C. Gregory were provided by the INTA (Instituto Nacional de Tecnología Agropecuaria) peanut germplasm bank of Manfredi, Córdoba, Argentina. All species used in this work were cultivated in the same crop year (1993/1994), season and place (INTA, Manfredi). All seeds had the same conditions for decreasing environment effects in the 
result expected. The collection data and classification is presented in the Table I. A detailed description of the taxonomic classification of peanut was reported by Krapovickas and Gregory (1994). Maturity of seed was establish when they showed maximum dry weight and maximum viability (Crookston and Hill, 1978).

\section{Determination of oil, ash, protein, moisture, and carbodydrate contents}

Three samples each containing five seeds from each cultivar were examined for oil, protein, ash and moisture contents. These seeds were selected at random. The sampling size was previously calculated (Cochran, 1974) and it was proper to the statistical design.
Seeds were milled and oil was extracted for $16 \mathrm{~h}$ with petroleum ether (boiling range $30-60^{\circ} \mathrm{C}$ ) in a Soxhlet apparatus. The extracted oils were dried over anhydrous sodium sulfate and the solvent removed under reduced pressure in a rotary film evaporator. Oil percentages was determined by weight difference.

Moisture, ash, nitrogen contents were determined according to AOAC (1980). Ash was performed by incineration in a muffle furnace at $525^{\circ} \mathrm{C}$. The nitrogen content was estimated by the Kjeldahl method and converted to protein percentage by using the conversion factor 5,46 (Young and Hammons, 1973). Carbohydrate content was estimated by value difference of the other components (expressed on dry basis) using the formula: carbohydrate content $=100 \%$ $-(\%$ protein $+\%$ oil $+\%$ ash $)$.

Table I

Collection data of wild species of Arachis from Argentina (Arg) and Bolivia (Bol)

\begin{tabular}{|c|c|c|c|}
\hline $\begin{array}{l}\text { Lot } \\
89 / 90 \text { a }\end{array}$ & Species & $\begin{array}{c}\text { Collection } \\
\text { numberb }\end{array}$ & Location \\
\hline 1 & A. correntina & K 7897 & $10 \mathrm{Km}$ west or Ruta 12,. Ituzaingó, Corrientes, Arg. \\
\hline 2 & A. correntina & GKP 9530-31 & Ruta 12, Arroyo Riachuelo, Corrientes, Arg. \\
\hline 3 & A. correntina & GKP 9548 & Ramada Paso, Corrientes, Arg. \\
\hline 4 & A. durannensis & K 7988 & Campo Durán, Salta, Arg. \\
\hline 5 & A. durannensis & K 7988-2 & Campo Durán, Salta, Arg. \\
\hline 6 & A. durannensis & K 7988-3 & Campo Durán, Salta, Arg. \\
\hline 7 & A. durannensis & KGBPScS 30060 & Río Perico, El Carmen, Jujuy, Arg. \\
\hline 8 & A. durannensis & KGBPScS 30061 & Palpalá, Jujuy, Arg. \\
\hline 9 & A. durannensis & KGBPScS 30064 & San Salvador (Ruta 66), Jujuy, Arg. \\
\hline 10 & A. durannensis & KGBPScS 30065 & Senda Hachada, Salta, Arg. \\
\hline 11 & A. durannensis & KGBPScS 30067 & Ruta 34 and Río Seco, Senda Hachada, Salta, Arg. \\
\hline 12 & A. durannensis & KGBPScS 30069 & $30 \mathrm{Km}$ north of Yacuiba, Caiza, Tarija, Bol. \\
\hline 13 & A. durannensis & KGBPScS 30070 & $18 \mathrm{Km}$ north of Yacuiba, Caiza, Tarija, Bol. \\
\hline 14 & A. durannensis & KGBPScS 30073 & $30 \mathrm{Km}$ north of Tarija, Bol. \\
\hline 15 & A. durannensis & KGBPScS 30071 & $2 \mathrm{Km}$ west of Saladillo, Tarija, Bol. \\
\hline 16 & A. durannensis & KGBPScS 30072 & $14 \mathrm{Km}$ west of Caraparí, Tarija, Bol. \\
\hline 17 & A. durannensis & KGBPScS 30074 & $2 \mathrm{Km}$ north of Palmar Grande, Santa Cruz, Bol. \\
\hline 18 & A. durannensis & KSBScC 36002 & Río Juramento, El Tunal, Salta, Bol. \\
\hline 19 & A. durannensis & KSBScC 36004 & Palpalá, Jujuy, Arg. \\
\hline 22 & A. durannensis & KGP 10038-2 & El Prado, Río Arenales, Salta, Arg. \\
\hline 23 & A. durannensis & KScGo 28458 & Campo Durán, Salta, Arg. \\
\hline 24 & A. monticola & BaRiK 7264-1 & Yala, Jujuy, Arg. \\
\hline 26 & A. monticola & KGBPScS 30063 & Lozano, Jujuy, Arg. \\
\hline 27 & A. batizocoi & K 9484 & Río Parapetí, Bol. \\
\hline 28 & A. batizocoi & KGBPScS 30079 & Paja Colorada, Bol. \\
\hline 29 & A. batizocoi & KGBPScC 30081 & Lagunillas, Santa Cruz, Bol. \\
\hline 30 & A. batizocoi & KGBPScS 30083 & Gutiérrez, Santa Cruz, Bol. \\
\hline 35 & A. cardenasii & KSSc 36015 & Roboré, Santa Cruz, Bol. \\
\hline 36 & A. cardenasii & KSSc 36016 & Roboré, Santa Cruz, Bol. \\
\hline 37 & A. cardenasii & KSSc 36020 & Roboré, Santa Cruz, Bol. \\
\hline 38 & A. cardenasii & KSSc 36021 & Santiago, Bol. \\
\hline 39 & A. cardenasii & KSSc 36033 & Natividad, Santa Cruz, Bol. \\
\hline 40 & A. cardenasii & KSSc 36034 & Natividad, Santa Cruz, Bol. \\
\hline
\end{tabular}

a Number of lot in INTA of Manfredi, Córdoba, Argentina.

b Voucher specimens have been deposited at Museo Botánico de Corrientes (CTES), Argentina. 


\section{Fatty Acid Composition}

Fatty acid methyl esters were prepared by transmethylation with a $3 \%$ solution of sulfuric acid in methanol, as previously described (Jellum and Worthington, 1966). The fatty acid methyl esters of total lipids were analyzed on a Shimadzu GC-R1A gas chromatograph equipped with flame ionization detector (FID). AT-WAX superox II capillary columm (30 $\mathrm{m} \times 0.25 \mathrm{~mm}$ i.d.) was used. Column temperature was programmed from $180^{\circ} \mathrm{C}$ (held for $10 \mathrm{~min}$ ) to $240^{\circ} \mathrm{C}\left(4^{\circ} \mathrm{C} / \mathrm{min}\right)$. Injector temperature was $250^{\circ} \mathrm{C}$. The carrier (nitrogen) had a flow rate of $1 \mathrm{~mL} / \mathrm{min}$. The separated fatty acid methyl esters were identified by comparing their retention times with those of authentic samples wich were purchased from SIGMA Chemical Co. Quantitative analysis of the fatty acids were performed using the heptadecanoic acid methyl ester as internal standard. lodine values were calculated from fatty acid composition (Hashim et al., 1993) using the formula: I.V. $=(\%$ oleic $\times 0.8601)+(\%$ linoleic $x$ $1.7321)+(\%$ eicosenoic $\times 0.7854)$.

\section{Sterol Composition}

Sterols of the unsaponifiable matter from $5 \mathrm{~g}$ of oil (after saponification with alcoholic $1 \mathrm{M}$ potassium hydroxide) were purified by preparative thin-layer chromatography (TLC). TLC was performed on silica gel $60 \mathrm{G}(20 \times 20 \mathrm{~cm}, 0,5 \mathrm{~mm}$ layer thickness) plates using chloroform-diethyl ether $(9: 1 \mathrm{v} / \mathrm{v})$ as the developing solvent. The approximate relative $R_{f}$ values of the 4-desmethylsterols fraction was 0,27 . The unsaponifiable matter was dissolved in chloroform (5\%) and $150 \mu \mathrm{l}$ was deposited as a streak of $15 \mathrm{~cm}$ length on the plate. Cholesterol, used as standard, was spotted on the left and right hand sides of the plate. The corresponding band of 4-desmethylsterols was scraped off the plate and extracted with chloroform (Gaydou et al., 1983). Purified sterols were analyzed on a Shimadzu GC-R1A gas chromatograph equipped with FID. Shimadzu CBP1 capillary column $(25 \mathrm{~m} \times$ $0,25 \mathrm{~mm}$ i.d.) was used. Column temperature was programmed from 200 to $300{ }^{\circ} \mathrm{C}\left(4^{\circ} \mathrm{C} / \mathrm{min}\right)$. Injector temperature was $320^{\circ} \mathrm{C}$. The carrier (nitrogen) had a flow rate of $1 \mathrm{~mL} / \mathrm{min}$. Standard sterols (Sigma Chemical Co.) were run in order to use retention times in identifying sample peaks. The amount of sterols was determined from the weight of $5 a$-cholestane as internal standard. The data were calculated using a integrator of the chromatograph.

\section{Statistical analysis}

All analysis for each sample of the wild Arachis were done in triplicate. Mean and standard deviation for each species were determined and significant differences among mean values were evaluated using a t-test (Branch et al., 1990) due the data shown normal distribution according previously researchs performed in this material. Mean values of $A$. monticola were only two genotypes and was not included in the statistical analysis.

\section{RESULTS AND DISCUSSION}

Moisture, oil, protein, ash, and carbohydrate contents and iodine value are shown in Table II. Peanuts are characterized by high oil and protein contents and low carbohydrates and ash. Knowledge of these components is important in the end products of the industry (Ahmed and Young, 1982). The oil content was highest in $A$. batizocoi (mean value $53,35 \%)$ Arachis monticola and $A$. durannensis exhibited the highest protein levels.

lodine value and $\mathrm{O} / \mathrm{L}$ ratio are both indicators of peanut oil stability and shelf-life (Ahmed and Young, 1982). Higher O/L ratios and lower iodine values suggest better stability, longer shelf-life, and quality of the oils (Branch et al., 1990). All wild species of peanut had in relation to iodine value and $O / L$ ratio, lower stability and quality of their oils than the US peanut cultivars (Branch et al., 1990) and Argentina (Grosso et al., 1994). These variations could be due to differences in climatic conditions, soil moisture and air temperature during maturation of peanut seed. The best $\mathrm{O} / \mathrm{L}$ ratio and iodine values were found in $A$. correntina (mean value 1,06 ) and $A$. batizocoi (mean value 106), respectively (Table II).

The fatty acid composition is presented in Table III. Palmitic (16:0), stearic $(18: 0)$, oleic $(18: 1)$, linoleic (18:2), arachidic (20:0), eicosenoic (20:1), behenic $(22: 0)$ and lignoceric (24:0) acids were detected. Oleic and linoleic acids were the principal components (Table III). Oleic acid was predominant in the samples of $A$. correntina. The lowest oleic and highest linoleic concentrations were the principal differences with respect to the fatty acid composition of peanut cultivars previously published (Branch et al., 1990; Grosso and Guzmán, 1995a) and with other species of Arachis (Stalker et al., 1989). The differences between species detected in this work are not probably due to climatic conditions, because all sample of especies were cultivated in the same year, growing season and locality. However, the differences observed with data previously reported could be due these causes.

The sterols are components of unsaponifiable lipids and are important to identify blends of fats and oils (Belitz and Grosch, 1987). Tocopherols and sterols are also of interest because of their antioxidant activity (Dutta and Appelqvist, 1996). The total sterols in peanut are about $0,5 \%$ of oil (Formo et al., 1979). The following sterols were detected (Table IV): cholesterol, campesterol, stigmasterol, B-sitosterol, $\Delta^{5}$ avenasterol, $\Delta^{7}$-stigmasterol, and $\Delta^{7}$-avenasterol. Arachis correntina showed higher concentrations of campesterol $(16,47 \%)$, stigmasterol $(12,27 \%)$ and $\Delta^{5}$ avenasterol, (12,13\%) and lower B-sitosterol $(56,40 \%)$ than the other species. These results shown similarity with peanut cultivars previously reported (Grosso and Guzmán, 1995b). Some significant differences were found in sterols. 
Table II

Moisture, oil, protein, ash, and carbohydrate (Carb.) contents (wt\%) and iodine value of wild peanuts from Argentina and Bolivia

\begin{tabular}{|c|c|c|c|c|c|c|c|}
\hline \multicolumn{2}{|c|}{ Species } & \multirow{2}{*}{$\frac{\text { Moisture }}{5.5}$} & \multirow{2}{*}{$\frac{\text { Oila }}{50.3}$} & \multirow{2}{*}{$\begin{array}{l}\text { Proteina } \\
27.5\end{array}$} & \multirow{2}{*}{$\frac{\text { Ash }^{\mathrm{a}}}{2.4}$} & \multirow{2}{*}{$\frac{\text { Carba }}{19.8}$} & \multirow{2}{*}{$\frac{\text { I.V. }}{105}$} \\
\hline 1 & A. correntina & & & & & & \\
\hline 2 & A. correntina & 5.9 & 51.5 & 26.5 & 2.7 & 19.3 & 109 \\
\hline \multirow[t]{3}{*}{3} & A. correntina & 5.7 & 49.5 & 26.1 & 2.5 & 21.9 & 108 \\
\hline & $M(n=3)^{b}$ & $5.70 \mathrm{a}$ & $50.43 a b$ & $26.70 \mathrm{~b}$ & $2.53 a$ & $20.33 a$ & $107.3 a$ \\
\hline & SD & \pm 0.20 & \pm 1.01 & \pm 0.72 & \pm 0.15 & \pm 1.43 & \pm 2.1 \\
\hline 4 & A. durannensis & 5.5 & 52.9 & 30.5 & 2.7 & 13.9 & 110 \\
\hline 5 & A. durannensis & 5.8 & 52.5 & 29.3 & 2.5 & 15.7 & 109 \\
\hline 6 & A. durannensis & 5.8 & 51.7 & 31.7 & 2.3 & 14.3 & 111 \\
\hline 7 & A. durannensis & 5.9 & 51.5 & 27.3 & 2.8 & 18.4 & 113 \\
\hline 8 & A. durannensis & 5.6 & 53.2 & 27.5 & 2.6 & 16.7 & 113 \\
\hline 9 & A. durannensis & 5.7 & 51.2 & 26.9 & 2.6 & 14.3 & 110 \\
\hline 10 & A. durannensis & 5.5 & 52.2 & 28.3 & 2.4 & 17.1 & 107 \\
\hline 11 & A. durannensis & 5.9 & 53.0 & 29.6 & 2.9 & 14.5 & 109 \\
\hline 12 & A. durannensis & 5.3 & 53.8 & 31.9 & 2.5 & 11.8 & 110 \\
\hline 13 & A. durannensis & 5.5 & 52.3 & 30.6 & 2.5 & 14.6 & 110 \\
\hline 14 & A. durannensis & 5.5 & 49.6 & 30.8 & 2.6 & 17.0 & 109 \\
\hline 15 & A. durannensis & 5.7 & 53.6 & 29.4 & 2.7 & 14.3 & 108 \\
\hline 16 & A. durannensis & 5.8 & 49.6 & 28.2 & 2.3 & 19.9 & 108 \\
\hline 17 & A. durannensis & 5.5 & 53.4 & 30.5 & 2.4 & 13.7 & 112 \\
\hline 18 & A. durannensis & 5.6 & 49.2 & 28.7 & 2.7 & 19.4 & 108 \\
\hline 19 & A. durannensis & 5.6 & 49.6 & 28.7 & 2.8 & 18.9 & 112 \\
\hline 22 & A. durannensis & 5.7 & 53.7 & 27.5 & 2.6 & 16.2 & 110 \\
\hline \multirow[t]{3}{*}{23} & A. durannensis & 5.6 & 49.9 & 27.0 & 2.6 & 20.5 & 110 \\
\hline & $M(n=18)^{b}$ & $5.64 a$ & $51.83 b$ & $29.13 c$ & $2.58 \mathrm{a}$ & $16.17 b$ & $109.9 b$ \\
\hline & SD & \pm 0.16 & \pm 1.61 & \pm 1.60 & \pm 0.17 & \pm 2.48 & \pm 1.7 \\
\hline 24 & A. monticola & 5.8 & 50.1 & 28.2 & 2.4 & 19.3 & 109 \\
\hline \multirow[t]{3}{*}{26} & A. monticola & 5.5 & 49.0 & 30.6 & 2.7 & 17.7 & 105 \\
\hline & $M(n=2)$ & 5.65 & 49.55 & 29.40 & 2.55 & 18.50 & 107.0 \\
\hline & $S D$ & \pm 0.21 & \pm 0.78 & \pm 1.70 & \pm 0.21 & \pm 0.91 & \pm 2.8 \\
\hline 27 & A. batizocoi & 5.5 & 53.2 & 28.8 & 2.5 & 15.5 & 105 \\
\hline 28 & A. batizocoi & 5.4 & 52.6 & 26.7 & 2.4 & 18.3 & 109 \\
\hline 29 & A. batizocoi & 5.8 & 53.7 & 27.6 & 2.7 & 16.0 & 106 \\
\hline \multirow[t]{3}{*}{30} & A. batizocoi & 5.5 & 53.9 & 25.2 & 2.6 & 18.3 & 104 \\
\hline & $M(n=4)^{b}$ & $5.55 a$ & $53.35 c$ & $27.07 a b$ & $2.55 a$ & $17.02 b$ & $106.0 \mathrm{a}$ \\
\hline & SD & \pm 0.17 & \pm 0.58 & \pm 1.52 & \pm 0.13 & \pm 1.59 & \pm 2.2 \\
\hline 35 & A. cardenasii & 5.9 & 51.7 & 28.5 & 2.6 & 17.2 & 104 \\
\hline 36 & A. cardenasii & 5.5 & 51.0 & 27.9 & 2.6 & 18.5 & 104 \\
\hline 37 & A. cardenasii & 5.5 & 50.3 & 28.2 & 2.4 & 19.1 & 107 \\
\hline 38 & A. cardenasii & 5.7 & 48.9 & 26.3 & 2.8 & 22.0 & 109 \\
\hline 39 & A. cardenasii & 5.8 & 49.0 & 26.6 & 2.6 & 21.8 & 108 \\
\hline \multirow[t]{3}{*}{40} & A. cardenasii & 5.6 & 51.6 & 27.5 & 2.5 & 18.4 & 108 \\
\hline & $M(n=6)^{b}$ & $5.67 a$ & $50.42 a$ & $27.50 \mathrm{a}$ & $2.58 \mathrm{a}$ & 19.50a & $106.7 \mathrm{a}$ \\
\hline & SD & \pm 0.16 & \pm 1.24 & \pm 0.88 & \pm 0.13 & \pm 1.95 & \pm 2.2 \\
\hline
\end{tabular}

\footnotetext{
a Expressed on dry weight basis.
}

b Mean values (M) and standard deviations (SD) for each species. Means followed by the same letter within each column are not significantly different at $\mathrm{P}=0.05$. 
Table III

Fatty acid composition (wt\%) of wild peanuts from Argentina and Bolivia

\begin{tabular}{|c|c|c|c|c|c|c|c|c|c|c|}
\hline & cies & $16: 0$ & 18:0 & $18: 1$ & $18: 2$ & 20:0 & $20: 1$ & 22:0 & $24: 0$ & O/L \\
\hline 1 & A. correntina & 9.9 & 1.8 & 43.3 & 38.7 & 1.1 & 1.4 & 2.5 & 1.1 & 1.22 \\
\hline 2 & A. correntina & 10.1 & 1.4 & 41.5 & 42.0 & 0.7 & 1.1 & 2.2 & 0.5 & 0.99 \\
\hline \multirow[t]{3}{*}{3} & A. correntina & 9.7 & 1.7 & 43.0 & 40.0 & 1.2 & 1.1 & 2.2 & 0.7 & 1.07 \\
\hline & $M(n=3)^{a}$ & $9.90 \mathrm{a}$ & $1.63 a$ & $42.60 c$ & $40.23 a$ & $1.00 \mathrm{a}$ & $1.20 \mathrm{a}$ & $2.30 \mathrm{~b}$ & $0.77 \mathrm{~b}$ & $1.06 c$ \\
\hline & SD & \pm 0.20 & \pm 0.21 & \pm 0.96 & \pm 1.66 & \pm 0.26 & \pm 0.17 & \pm 0.17 & \pm 0.31 & \pm 0.07 \\
\hline 4 & A. durannensis & 10.4 & 1.4 & 34.2 & 45.9 & 1.3 & 1.4 & 3.4 & 1.6 & 0.74 \\
\hline 5 & A. durannensis & 10.5 & 1.8 & 34.8 & 44.9 & 1.3 & 1.5 & 3.5 & 1.5 & 0.77 \\
\hline 6 & A. durannensis & 10.0 & 1.4 & 33.7 & 46.8 & 1.0 & 1.7 & 3.5 & 1.5 & 0.72 \\
\hline 7 & A. durannensis & 9.9 & 1.9 & 33.5 & 48.0 & 0.9 & 1.4 & 2.8 & 1.2 & 0.70 \\
\hline 8 & A. durannensis & 9.7 & 2.5 & 34.0 & 47.9 & 0.9 & 1.5 & 2.6 & 0.6 & 0.71 \\
\hline 9 & A. durannensis & 10.4 & 1.6 & 37.3 & 44.4 & 0.8 & 1.6 & 2.8 & 0.8 & 0.84 \\
\hline 10 & A. durannensis & 11.0 & 1.5 & 35.9 & 43.2 & 1.6 & 1.7 & 3.5 & 1.3 & 0.83 \\
\hline 11 & A. durannensis & 11.0 & 1.9 & 36.9 & 44.2 & 1.2 & 1.2 & 2.5 & 1.0 & 0.83 \\
\hline 12 & A. durannensis & 10.3 & 2.1 & 35.2 & 45.5 & 1.0 & 1.1 & 3.1 & 1.3 & 0.77 \\
\hline 13 & A. durannensis & 10.1 & 2.5 & 33.1 & 46.9 & 1.3 & 1.0 & 2.9 & 1.3 & 0.70 \\
\hline 14 & A. durannensis & 9.5 & 3.0 & 37.0 & 44.3 & 1.5 & 1.0 & 2.9 & 0.7 & 0.83 \\
\hline 15 & A. durannensis & 11.5 & 2.4 & 34.3 & 44.4 & 1.6 & 1.5 & 3.2 & 0.8 & 0.77 \\
\hline 16 & A. durannensis & 9.7 & 2.3 & 37.3 & 43.5 & 1.2 & 1.4 & 2.9 & 1.3 & 0.86 \\
\hline 17 & A. durannensis & 9.8 & 1.4 & 33.4 & 47.6 & 0.9 & 1.0 & 3.8 & 1.5 & 0.70 \\
\hline 18 & A. durannensis & 11.9 & 1.0 & 36.2 & 47.6 & 1.2 & 1.1 & 3.3 & 1.2 & 0.83 \\
\hline 19 & A. durannensis & 9.4 & 2.2 & 33.2 & 48.6 & 1.2 & 1.4 & 3.1 & 1.3 & 0.70 \\
\hline 22 & A. durannensis & 11.1 & 2.5 & 34.6 & 45.9 & 1.2 & 1.0 & 2.6 & 1.0 & 0.75 \\
\hline \multirow[t]{3}{*}{23} & A. durannensis & 10.1 & 2.3 & 33.8 & 45.9 & 1.3 & 1.4 & 3.2 & 1.4 & 0.74 \\
\hline & $M(n=18)^{a}$ & $10.35 a$ & $1.98 a$ & $34.91 \mathrm{a}$ & $45.86 b$ & $1.19 a$ & $1.33 a$ & $3.09 a$ & $1.18 a$ & $0.77 a$ \\
\hline & SD & \pm 0.70 & \pm 0.52 & \pm 1.48 & \pm 1.67 & \pm 0.24 & \pm 0.24 & \pm 0.37 & \pm 0.30 & \pm 0.06 \\
\hline 24 & A. monticola & 10.6 & 2.4 & 38.3 & 43.5 & 1.1 & 1.1 & 2.2 & 0.6 & 0.88 \\
\hline \multirow[t]{3}{*}{26} & A. monticola & 10.7 & 2.8 & 37.6 & 41.4 & 1.7 & 1.6 & 2.3 & 1.3 & 0.91 \\
\hline & $M(n=2)$ & 10.65 & 2.60 & 37.95 & 42.45 & 1.40 & 1.35 & 2.25 & 0.95 & 0.89 \\
\hline & SD & \pm 0.07 & \pm 0.28 & \pm 0.49 & \pm 1.48 & \pm 0.42 & \pm 0.35 & \pm 0.07 & \pm 0.49 & \pm 0.02 \\
\hline 27 & A. batizocoi & 10.7 & 1.5 & 38.5 & 40.9 & 1.5 & 1.8 & 3.2 & 1.5 & 0.94 \\
\hline 28 & A. batizocoi & 10.4 & 2.2 & 41.8 & 41.3 & 0.8 & 1.5 & 1.2 & 0.5 & 1.01 \\
\hline 29 & A. batizocoi & 12.7 & 2.3 & 37.4 & 42.2 & 1.1 & 0.9 & 2.1 & 0.8 & 0.89 \\
\hline \multirow[t]{3}{*}{30} & A. batizocoi & 10.6 & 2.2 & 39.7 & 39.8 & 1.2 & 1.4 & 3.6 & 1.0 & 1.00 \\
\hline & $M(n=4)^{a}$ & 11.10b & $2.05 a$ & 39.35b & $41.05 a$ & $1.15 a$ & 1.40ab & $2.52 b$ & $0.95 a b$ & $0.96 \mathrm{~b}$ \\
\hline & SD & \pm 1.07 & \pm 0.37 & \pm 1.88 & \pm 0.99 & \pm 0.29 & \pm 0.37 & \pm 1.09 & \pm 0.42 & \pm 0.06 \\
\hline 35 & A. cardenasii & 11.6 & 1.7 & 37.0 & 40.7 & 1.2 & 1.7 & 3.8 & 1.6 & 0.91 \\
\hline 36 & A. cardenasii & 10.8 & 1.6 & 35.9 & 41.2 & 1.9 & 2.6 & 3.6 & 1.8 & 0.87 \\
\hline 37 & A. cardenasii & 11.6 & 1.9 & 34.5 & 44.3 & 1.2 & 1.5 & 3.5 & 1.2 & 0.78 \\
\hline 38 & A. cardenasii & 10.7 & 1.4 & 36.4 & 44.5 & 0.9 & 1.6 & 3.3 & 1.2 & 0.82 \\
\hline 39 & A. cardenasii & 11.3 & 1.2 & 32.7 & 45.6 & 1.8 & 1.2 & 4.3 & 1.4 & 0.71 \\
\hline \multirow[t]{3}{*}{40} & A. cardenasii & 11.3 & 2.5 & 33.0 & 45.1 & 0.9 & 1.6 & 3.7 & 1.4 & 0.73 \\
\hline & $M(n=6)^{a}$ & $11.22 b$ & $1.72 a$ & $34.91 a$ & 43.57c & $1.32 a$ & $1.70 b$ & $3.70 \mathrm{c}$ & $1.43 c$ & $0.80 a$ \\
\hline & SD & \pm 0.39 & \pm 0.45 & \pm 1.80 & \pm 2.08 & \pm 0.44 & \pm 0.47 & \pm 0.34 & \pm 0.23 & \pm 0.08 \\
\hline
\end{tabular}

a Mean values (M) and standard deviations (SD) for each species. Means followed by the same letter within each column are not significantly different at $\mathrm{P}=0.05$. 
Table IV

Sterol composition (wt\%) of wild peanuts from Argentina and Bolivia

\begin{tabular}{|c|c|c|c|c|c|c|c|c|}
\hline \multicolumn{2}{|c|}{ Species } & \multirow{2}{*}{$\frac{\text { Chol. }}{1.3}$} & \multirow{2}{*}{$\frac{\text { Camp. }}{16.4}$} & \multirow{2}{*}{$\begin{array}{l}\text { Stig. } \\
12.8\end{array}$} & \multirow{2}{*}{$\begin{array}{l}\text { Sit. } \\
55.6\end{array}$} & \multirow{2}{*}{$\frac{\Delta^{5} \text {-av. }}{11.6}$} & \multirow{2}{*}{$\frac{\Delta^{7} \text {-stig. }}{0.9}$} & \multirow{2}{*}{$\frac{\Delta^{7} \text {-av. }}{1.1}$} \\
\hline 1 & A. correntina & & & & & & & \\
\hline 2 & A. correntina & 0.9 & 15.9 & 12.6 & 56.2 & 12.3 & 1.2 & 0.7 \\
\hline \multirow[t]{3}{*}{3} & A. correntina & 2.0 & 17.1 & 11.4 & 57.4 & 12.5 & 1.5 & 1.5 \\
\hline & $M(n=3)^{b}$ & $1.40 \mathrm{a}$ & $16.47 b$ & $12.27 b$ & $56.40 b$ & $12.13 b$ & $1.20 \mathrm{~b}$ & $1.10 \mathrm{a}$ \\
\hline & SD & \pm 0.56 & \pm 0.60 & \pm 0.76 & \pm 0.92 & \pm 0.47 & \pm 0.30 & \pm 0.40 \\
\hline 4 & A. durannensis & 0.6 & 15.2 & 13.0 & 59.7 & 9.4 & 0.6 & 1.3 \\
\hline 5 & A. durannensis & 1.8 & 16.8 & 10.9 & 56.7 & 12.3 & 0.3 & 1.1 \\
\hline 6 & A. durannensis & 2.3 & 14.1 & 12.3 & 58.6 & 10.0 & 1.7 & 0.8 \\
\hline 7 & A. durannensis & 1.5 & 16.1 & 9.8 & 60.0 & 8.8 & 2.0 & 1.7 \\
\hline 8 & A. durannensis & 2.1 & 13.0 & 12.5 & 59.5 & 11.5 & 0.9 & 0.3 \\
\hline 9 & A. durannensis & 1.0 & 17.2 & 10.3 & 59.7 & 9.7 & 0.9 & 1.0 \\
\hline 10 & A. durannensis & 2.7 & 15.1 & 12.0 & 55.2 & 12.3 & 1.4 & 1.0 \\
\hline 11 & A. durannensis & 2.1 & 14.9 & 11.3 & 56.4 & 11.5 & 1.7 & 2.0 \\
\hline 12 & A. durannensis & 1.7 & 16.0 & 12.1 & 57.5 & 10.7 & 0.9 & 0.8 \\
\hline 13 & A. durannensis & 2.3 & 14.8 & 10.9 & 57.1 & 12.0 & 1.2 & 1.7 \\
\hline 14 & A. durannensis & 0.6 & 16.0 & 11.8 & 59.1 & 11.0 & 0.7 & 0.6 \\
\hline 15 & A. durannensis & 1.8 & 15.3 & 9.6 & 59.8 & 10.7 & 1.3 & 1.2 \\
\hline 16 & A. durannensis & 2.0 & 17.1 & 11.0 & 58.9 & 8.6 & 0.7 & 1.5 \\
\hline 17 & A. durannensis & 0.8 & 16.3 & 10.1 & 58.6 & 11.0 & 1.7 & 1.3 \\
\hline 18 & A. durannensis & 1.6 & 15.9 & 12.0 & 58.2 & 10.9 & 0.3 & 0.8 \\
\hline 19 & A. durannensis & 1.9 & 16.7 & 10.3 & 55.0 & 12.7 & 1.6 & 1.3 \\
\hline 22 & A. durannensis & 2.4 & 13.5 & 11.6 & 56.6 & 11.7 & 2.0 & 1.6 \\
\hline \multirow[t]{3}{*}{23} & A. durannensis & 1.6 & 15.8 & 12.3 & 57.1 & 10.1 & 1.7 & 1.3 \\
\hline & $M(n=18)^{b}$ & $1.71 \mathrm{a}$ & $15.54 a b$ & 11.32ab & $57.98 a$ & $10.83 a$ & $1.20 \mathrm{ab}$ & $1.18 \mathrm{a}$ \\
\hline & $S D$ & \pm 0.62 & \pm 1.18 & \pm 1.01 & \pm 1.59 & \pm 1.20 & \pm 0.60 & \pm 0.46 \\
\hline 24 & A. monticola & 2.1 & 15.6 & 12.3 & 56.1 & 10.7 & 1.4 & 1.6 \\
\hline \multirow[t]{3}{*}{26} & A. monticola & 1.7 & 16.0 & 11.4 & 55.3 & 11.7 & 1.9 & 1.5 \\
\hline & $M(n=2)$ & 1.90 & 15.80 & 11.85 & 55.70 & 11.20 & 1.65 & 1.55 \\
\hline & $S D$ & \pm 0.28 & \pm 0.28 & \pm 0.64 & \pm 0.57 & \pm 0.71 & \pm 0.35 & \pm 0.07 \\
\hline 27 & A. batizocoi & 2.0 & 15.2 & 9.7 & 61.6 & 10.0 & 0.9 & 0.3 \\
\hline 28 & A. batizocoi & 1.3 & 14.9 & 10.9 & 61.2 & 9.7 & 0.9 & 1.0 \\
\hline 29 & A. batizocoi & 0.9 & 16.8 & 11.3 & 55.7 & 12.0 & 1.8 & 1.3 \\
\hline \multirow[t]{3}{*}{30} & A. batizocoi & 1.9 & 16.1 & 10.8 & 56.3 & 11.5 & 1.5 & 1.3 \\
\hline & $M(n=4)^{b}$ & $1.52 \mathrm{a}$ & 15.75ab & $10.67 a$ & $58.70 a b$ & $10.80 a$ & $1.27 \mathrm{~b}$ & $0.97 a$ \\
\hline & SD & \pm 0.53 & \pm 0.87 & \pm 0.68 & \pm 3.13 & \pm 1.12 & \pm 0.45 & \pm 0.57 \\
\hline 35 & A. cardenasii & 1.5 & 13.7 & 10.5 & 59.4 & 12.0 & 1.5 & 0.9 \\
\hline 36 & A. cardenasii & 1.5 & 17.0 & 10.0 & 58.0 & 9.6 & 1.5 & 1.7 \\
\hline 37 & A. cardenasii & 2.3 & 14.9 & 11.4 & 60.1 & 10.7 & 0.6 & 0.3 \\
\hline 38 & A. cardenasii & 1.4 & 14.7 & 11.2 & 56.5 & 12.1 & 1.9 & 1.7 \\
\hline 39 & A. cardenasii & 0.9 & 15.3 & 11.7 & 57.2 & 12.0 & 1.3 & 1.2 \\
\hline \multirow{3}{*}{40} & A. cardenasii & 1.7 & 15.5 & 11.2 & 56.1 & 11.8 & 1.9 & 1.6 \\
\hline & $M(n=6)^{b}$ & $1.55 a$ & $15.18 a$ & $11.00 a$ & 57.88ab & $11.37 a b$ & $1.45 a b$ & $1.23 a$ \\
\hline & SD & \pm 0.45 & \pm 1.09 & \pm 0.63 & \pm 1.60 & \pm 1.01 & \pm 0.48 & \pm 0.63 \\
\hline
\end{tabular}

a Abbreviations: Chol. Cholesterol; Camp., Campesterol, Stig. Sitgmasterol, Sit; ß-sitosterol, $\Delta^{5}$-av., $\Delta^{5}$ avenasterol; $\Delta^{7}$-Sitg., $\Delta^{7}$ stigmasterol; $\Delta^{7}$-av. $\Delta^{7}$-avenasterol. tr.: less than $5 \mathrm{~g} / \mathrm{kg}$.

b Mean values (M) and standard deviations (SD) for each species. Means followed by the same letter within each column are not significantly different at $\mathrm{P}=0.05$. 
The chemical quality of oils (iodine value and $\mathrm{O} / \mathrm{L}$ ratio) from wild peanut studied in this work is not better than cultivated peanut. However, the chemical composition of wild species in Arachis contributes to characterization of germplasm bank materials.

\section{ACKNOWLEDGEMENTS}

This work was supported by CONICET, CONICOR, and SECYT. Antonio Krapovickas and José R. Pietrarelli identificated the samples. The plant material was provided by Agr. R. Sánchez.

\section{REFERENCES}

Ahmed, E. H. and Young, C. T. (1982). - «Composition, nutrition and flavor of peanut" en "Peanut Science and Technology".- H. E. Pattee and C. T. Young (Eds.). American Peanut Research and Education Society Inc., Yoakum, Texas, p. 655-687.

AOAC (1980). - «Official Methods of Analysis», 13th ed. Association of Official Analytical Chemists.- Washington DC.

Belitz, H. D. and Grosch, W. (1987). -«Food Chemistry», p. 187. -Springer Verlag, Berlin.

Branch, W. D., Nakayama, T. and Chinnan, M. S. (1990). - «Fatty acid variation among U. S. runner-type peanut cultivars".- J. Am. Oil Chem. Soc. 67, 591-593.

Cherry, J. P. (1977). - «Potential sources of peanut seed proteins and oil in the genus Arachis".- J. Agric. Food Chem. 25, 186-193.

Cochran, W. G. (1974). - "Técnicas de Muestreo».Compañía Editorial Continental, Mexico, p. 41-71.

Crookston, R. K. and Hill, D. S. (1978). - «Visual indicator of the physiological maturity of soybean seed".- Crop Sci. 18, 867-870.
Dutta, P. C. and Appelqvist, L. A. (1996). - «Sterols and sterol oxides in the potato products, and sterols in the vegetable oils used for industrial frying operations".- Grasas y Aceites 47, 38-47.

Formo, M. W., Jungermann, E., Norris, F. A. and Sonntag, N.O.V. (1979). - «Bailey's Industrial oil and fat products»4th ed. -D. Swern (Ed.). -John Wiley \& Sons, New York, p. 59-63.

Gaydou, E. M., Bianchini, J. P. and Ratovogery, J. (1983). - Triterpene alcohols, methyl sterols, sterols, and fatty acids in five Malagasy legume seed oils".- J. Agric. Food Chem. 31, 833-836.

Grosso, N. R. and Guzmán, C. A. (1995a). - Chemical characteristics of argentinian groundnut cultivars".International Arachis Newsletter 15, 17-18.

Grosso, N. R. and Guzmán, C. A. (1995b). - “Chemical composition of aboriginal peanut (Arachis hypogaea L.) seeds from Perú».- J. Agric. Food Chem. 43, 102-105.

Grosso, N. R. Lamarque, A. L., Maestri, D. M., Zygadlo, J. A. and Guzmán, C. A. (1994). - "Fatty acid variation of runner peanut (Arachis hypogaea L.) among geographic localities from Córdoba (Argentina)".- J. Am. Oil Chem. Soc. 71, 541-542.

Hashim, I. B., Koehler, P. E., Eitenmiller, R. R., Kvien, C. (1993). - «Fatty acid composition and tocopherol content of drought stressed Florunner peanuts".- Peanut Sci. 20, 21-24.

Jellum, M. D. and Worthington, R. E. (1966). -«A rapid method of fatty acid analysis of oil from individual corn (Zea mays L.) kernels".- Crop Sci. 6, 251-253.

Krapovickas, A. and Gregory, W. C. (1994). -«Taxonomía del género Arachis (Leguminosae)".- Bonplandia 8, 1-186.

Stalker, H. T., Young, C. T. and Jones, T. M. (1989). - «A survey on the fatty acids of peanut species".Oléagineux 44, 419-424.

Young, C. T. and Hammons, R. O. (1973). - «Variations in the protein levels of a wide range of peanut genotypes (Arachis hypogaea L.)".- Oleagineux 28, 293-297.

Recibido: Abril 1997 Aceptado: Julio 1997 